\title{
El papel de las mujeres amatlecas en la conservación biocultural dinámica del maíz nativo en Amatlán de Quetzalcóatl, Tepoztlán, Mor
}

Le rôle des femmes amatlecas dans la conservation bioculturelle dynamique du maïs natif dans Amatlán de Quetzalcoatl, Tepoztlán, Mor

Erica Hagman Aguilar and Montserrat Gispert Cruells

\section{OpenEdition}

\section{Journals}

\section{Electronic version}

URL: http://journals.openedition.org/ethnoecologie/3423

DOI: 10.4000/ethnoecologie.3423

ISSN: 2267-2419

Publisher

Laboratoire Eco-anthropologie et Ethnobiologie

\section{Electronic reference}

Erica Hagman Aguilar and Montserrat Gispert Cruells, «El papel de las mujeres amatlecas en la conservación biocultural dinámica del maíz nativo en Amatlán de Quetzalcóatl, Tepoztlán, Mor », Revue d'ethnoécologie [Online], 13 | 2018, Online since 25 June 2018, connection on 19 April 2019. URL : http://journals.openedition.org/ethnoecologie/3423; DOI : 10.4000/ethnoecologie.3423

This text was automatically generated on 19 April 2019

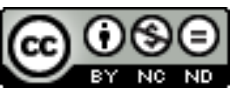

Revue d'ethnoécologie est mis à disposition selon les termes de la licence Creative Commons Attribution - Pas d'Utilisation Commerciale - Pas de Modification 4.0 International. 


\section{El papel de las mujeres amatlecas en la conservación biocultural dinámica del maíz nativo en Amatlán de Quetzalcóatl, Tepoztlán, Mor}

Le rôle des femmes amatlecas dans la conservation bioculturelle dynamique du maïs natif dans Amatlán de Quetzalcoatl, Tepoztlán, Mor

Erica Hagman Aguilar and Montserrat Gispert Cruells

\section{Introducción}

1 El cultivo de la milpa (Figura 1), integrado por la siembra de maíces (Zea mays L.), frijoles ( Phaseolus spp.) y calabazas (Cucurbita spp.) junto con el manejo de diferentes especies arvenses (varias familias, p. ej. Amaranthaceae, Chenopodiaceae, Solanaceae, Asteraceae y Fabaceae), no es una acción estática orientada únicamente al aprovechamiento de las especies biológicas que lo integran, sino que va acompañado de las cosmovisiones, las tradiciones, los rituales, las fiestas, las técnicas de manejo, las formas de aprovechamiento y la transformación de sus productos; en una labor dinámica que ha integrado de manera tradicional desde sus inicios, especies y variedades biológicas, formas y significados, marcados por sus contextos culturales, ambientales, socioeconómicos y políticos a lo largo de su historia. 
Figura 1 : Agroecosistema milpa en Amatlán de Quetzalcóatl, Morelos

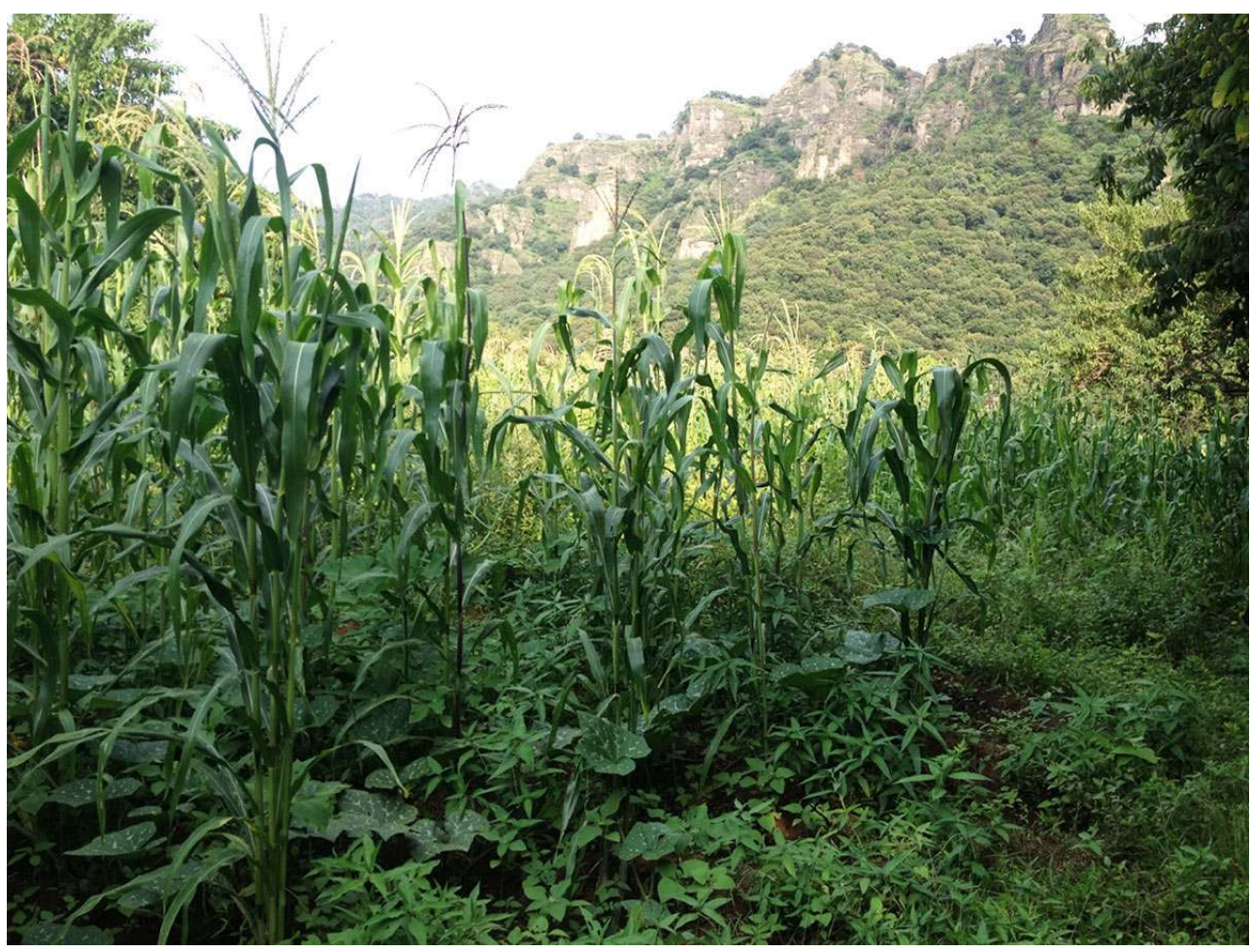

FOTOgRAFÍA DE E. L. HAgMAN AgUILAR

2 En particular, la gran riqueza genética del maíz existe gracias a que cientos de poblaciones locales nativas se siguen sembrando por diferentes razones (Ortega-Paczka 2003). La articulación de una estrategia nacional enfocada a la conservación in situ de la diversidad del maíz es de gran relevancia en nuestros días, abarcando no sólo el resguardo del germoplasma, sino también la preservación de los ecosistemas con sus interacciones bióticas y abióticas y los conocimientos entorno a ellos, salvaguardados por las comunidades que les dan sustento (Kato et al. 2013).

En la conservación biocultural dinámica del maíz, han participado históricamente hombres y mujeres por igual, cada uno contribuyendo desde su propio papel que se complementa mutuamente. Así, en una investigación tendiente a conocer este tema con mayor profundidad, es indispensable describir el papel de las mujeres, pues a pesar de su importancia en el ciclo de cultivo del maíz, existe poca documentación sobre el rol que han tenido en la conservación de los recursos fitogenéticos (FAO-IPGRI 2001).

4 El caso de la persistencia de la diversidad de maíz nativo y sus saberes en Amatlán de Quetzalcóatl, municipio de Tepoztlán, Morelos, es un ejemplo de conservación biocultural in situ. Sus actuales habitantes son de origen náhuatl, uno de los grupos étnicos que en el presente se resisten a perder sus tradiciones, cultura y linaje (Alvarado 1992, Corneli 2005) y se encuentran dentro de una región biodiversa que forma parte de dos sitios decretados como « Área de Protección de Flora y Fauna » y «Parque Nacional », que son respectivamente el Corredor Biológico Ajusco-Chichinautzin y El Tepozteco (Gispert et al. 2007).

5 El propósito del presente estudio fue exponer el papel de la mujer amatleca en la conservación del maíz nativo, inmerso en el manejo de su cultivo, en su proceso de 
diversificación y en las ceremonias alrededor de él; al mismo tiempo queremos mostrar la transformación culinaria del grano manifestada en la variedad de platillos en los que se ocupa de manera tradicional.

\section{Materiales y Métodos}

6 Amatlán de Quetzalcóatl es una de las seis ayudantías del municipio de Tepoztlán; el poblado está ubicado a siete kilómetros al este de la cabecera municipal, encontrándose el núcleo de viviendas entre las coordenadas $18^{\circ} 58^{\prime}$ de latitud norte y $99^{\circ} 02^{\prime}$ de longitud oeste, a una altitud de 1,634 m. s. n. del m. (INEGI 2010) (Figura 2). La asombrosa conjunción de componentes abióticos presentes en la región muestra su reflejo en la biodiversidad, siendo el sitio un punto de encuentro entre las especies de afinidad neártica y neotropical (López-García y Oliver-Guadarrama 2010) que exhibe una belleza escénica característica enmarcada por varios cerros que en su mayoría conservan su nombre en náhuatl.

Figura 2 : Localización y delimitación de Amatlán de Quetzalcóatl, Tepoztlán, Morelos. Realizado con shapefiles de la CONABIO (2012)

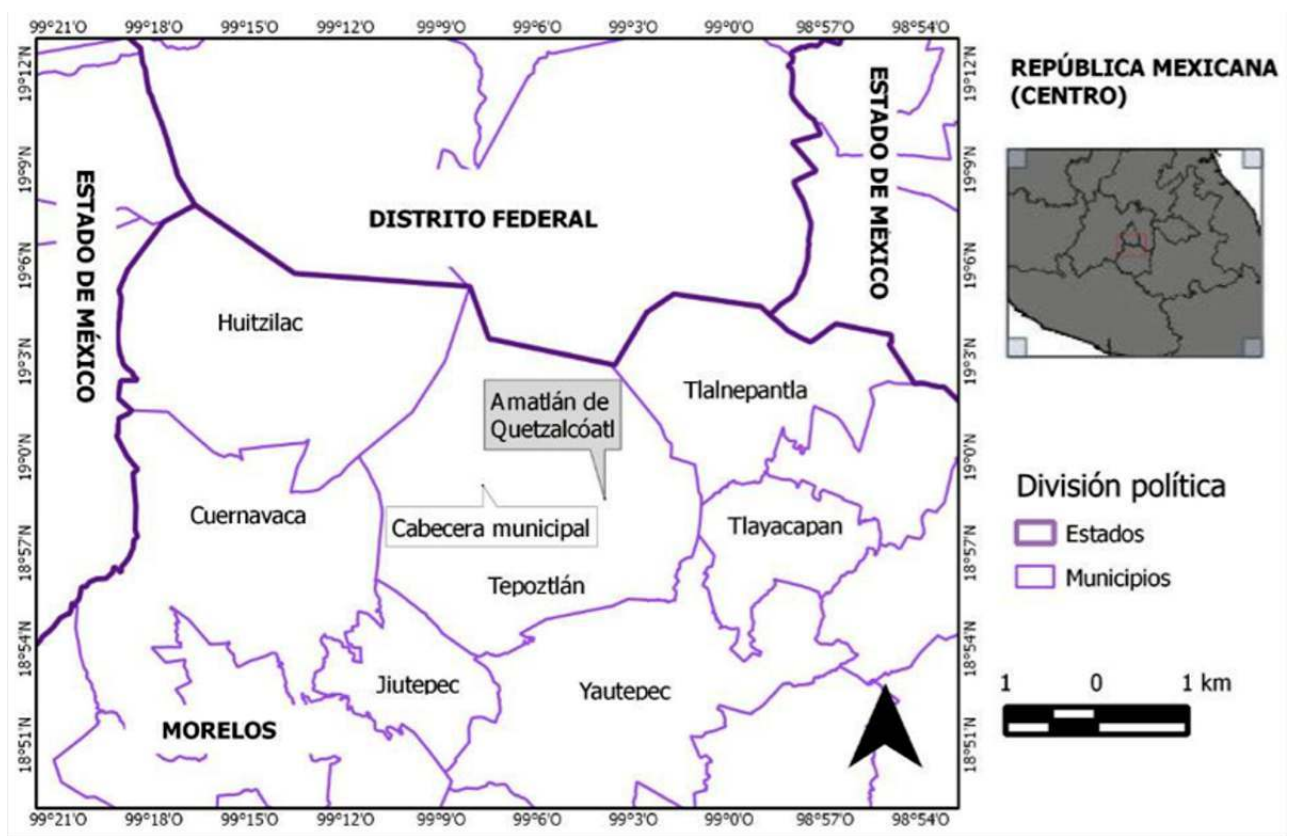

ELABORACIÓN PROPIA

7 Para llegar a nuestro propósito, utilizamos métodos cualitativos (interpretativo, comparativo e inductivo), lo que nos permitió reconocer las motivaciones de las mujeres que han propiciado la conservación del maíz nativo y las tradiciones relacionadas con él en Amatlán de Quetzalcóatl. Para la colecta y el análisis de los datos, empleamos varias técnicas que fueron de la mano en una interacción permanente, caracterizada por un proceso de acción-reflexión constante, es decir que el análisis de datos fue paralelo al acopio de los mismos (Bisquerra 1996).

Durante el trabajo de campo llevamos a cabo constantes visitas a lo largo de un año y medio, observando, aprendiendo, colaborando, trabajando y conviviendo con diferentes 
miembros de la comunidad, especialmente con las mujeres, que aportaron testimonios esenciales para el presente trabajo.

Ubicamos como expertas locales a cinco mujeres oriundas de Amatlán, mayores de 30 años, que participan en el proceso de cultivo (ciclo ritual y agrícola) y en la transformación culinaria del maíz nativo. Las técnicas de indagación que empleamos fueron individuales (Alexiades 1996) y ocurrieron tanto en las casas de las campesinas, como en recorridos por el campo y en sus milpas. Con cada una de las expertas realizamos una entrevista semi-estructurada (Gispert et al. 1979) y varias entrevistas informales y sin estructura (Alexiades 1996) con fines verificativos y para obtener información complementaria; todas ellas « en profundidad» (Taylor y Bogdan 1987). Para el registro de la información utilizamos grabaciones de audio, imágenes fotográficas y notas de campo.

Los resultados de las entrevistas fueron transcritos y ordenados por preguntas y por persona en una hoja de cálculo, de esa forma el contenido pudo ser categorizado posteriormente. Los resultados aquí presentes son el producto del análisis y la síntesis de los testimonios extraídos durante las entrevistas realizadas en el trabajo de campo, cotejando los datos con los registros hechos a través de la observación participante.

11 La observación participante como técnica interactiva (Rodríguez et al. 1999) se empleó antes y durante el estudio; a) desde la planificación, con el propósito de familiarizarnos con el contexto y con la comunidad, así como para desarrollar las directrices y las guías de las entrevistas; b) a lo largo del ciclo de cultivo, en las labores de la milpa y en las actividades de la vida cotidiana; y c) durante las visitas posteriores a la comunidad con fines verificativos.

\section{Resultados y discusión}

La información obtenida se muestra ordenada en cuatro categorías (A, B, C y D) correspondientes a las actividades, que consideramos, expresan el papel sustancial de las mujeres amatlecas en el mosaico cultural del sistema milpa, mostrado por las cinco expertas locales que participaron en el estudio, a las que presentamos a continuación:

1. La Sra. Amelia Escalante Zamora no trabaja en la milpa, pero si toma parte en las ceremonias, como en el recibimiento del maíz; desde niña su labor estuvo enfocada en las tareas hogareñas, entre ellas está elaborar y llevar los alimentos al campo para su esposo y sus colaboradores en la milpa.

2. La Sra. Dionisia Torres Ramírez empezó a ir al campo desde los 10 años, al «tlacolol » (del náhuatl tlacoloa: ir rodeando alguna parte) de su abuelo y se hizo cargo de la agricultura familiar en las ocasiones en que su esposo tuvo que emigrar a Canadá; en la actualidad, además de organizar a nivel familiar las ceremonias relacionadas con el maíz y preparar los alimentos, junto con sus hijas, su hijo y su esposo trabajan en la milpa.

3. La Sra. Sabina Casares Campos aprendió a trabajar en el campo desde los 15 años, junto con sus once hermanos y hermanas, al casarse ayudó a su marido en las labores del campo, aunque actualmente ya no interviene directamente como antes debido a su avanzada edad, pero sigue cocinando para su familia.

4. La Sra. Irene Ramírez Casares a los 17 años aprendió a sembrar con su padre; hoy en día se encarga de la cocina para su consumo y la venta de tortillas y guisados; y al ser soltera se ocupa igualmente de la milpa con el apoyo de familiares (principalmente sus sobrinos) o de peones. 
5. La Sra. Rosalinda Flores Corrales a la temprana edad de 8 años acompañaba a su padre, posteriormente cuando él murió, su madre y ella rentaban terrenos que sembraban para autoconsumo; ahora se encarga de guisar para su familia y trabajar en su milpa, con la ayuda limitada de su esposo.

En el estudio buscamos conocer y retransmitir el sentir y la cosmovisión de las participantes, por lo que mostramos la descripción detallada de la información analizada, junto con fragmentos fieles de las entrevistas que expresan la percepción de estas mujeres.

\section{El cultivo}

Las amatlecas intervienen de diversas formas en las prácticas agrícolas, en mayor o menor medida, sin descuidar sus tareas domésticas históricamente asignadas. Ya sea que se involucren de manera directa en la labor propia de cada etapa del ciclo o que se encarguen de la preparación de los alimentos y los lleven al campo, su papel es esencial en el proceso productivo del maíz.

15 En cuanto a las labores agrícolas tal cual, encontramos que: en el barbecho y en el surcado, la mujer amatleca puede ayudar a "uncir» los animales (atar o sujetar los bueyes al yugo); en la siembra, en la "primera mano" y en la "segunda mano", el hombre va adelante con la yunta y la mujer va atrás arrojando las semillas o el abono; por último, en la cosecha, las mujeres pocas veces ayudan a "pixcar» (del náhuatl pixca: cosechar las mazorcas), debido al arduo esfuerzo que ésta labor requiere.

16 Sin embargo, en todo el país cuando los hombres migran, las mujeres son las que se quedan y se convierten en las responsables de lo que ocurre con la tierra y en la tierra (COA 2012). Esto fue observado en el caso de las amatlecas que han continuado con el cultivo de la milpa cuando sus conyugues han migrado, para responder a las necesidades económicas familiares, manteniendo a su vez los valores culturales y sociales que ello implica. Román-Montes de Oca y Guzmán-Gómez (2013), destacan el lugar de la mujer amatleca en el campo, su participación productiva es amplia y fundamental, aunque se desenvuelve en un ámbito en el que se reconoce como algo extra, o como apoyo al trabajo principal que los hombres realizan.

17 Lo anterior denota que el trabajo en la milpa no es exclusivo del hombre, de hecho las sabedoras locales comentaron que no hay específicamente algo que se le prohíba hacer a la mujer en la milpa, aunque su participación depende de la posibilidad económica de contratar a algún peón, lo cual es poco frecuente.

\section{La selección de las semillas}

En el proceso de la selección anual de las semillas de maíz puede cooperar toda la familia, pero en el caso de que los hijos o nietos no puedan, lo hacen sólo el esposo y la esposa, ambos son los que lo desgranan, lo «curan » (lo bendicen y sahúman) y lo almacenan (Figura 3).

19 El rol activo de las mujeres en el proceso de selección de las semillas de maíz se basa principalmente en la búsqueda de características que favorezcan el rendimiento en cuanto al tamaño del grano. Sin embargo, es notable que las mujeres emplean variedades de semillas de diferentes colores en la elaboración de diversos platillos, como se verá más 
adelante, lo cual afecta su criterio al momento de la selección de las semillas que serán sembradas en el siguiente ciclo, interviniendo de manera directa en la preservación e incremento de la diversidad genética de los maíces nativos. Hecho también observado en el trabajo de la FAO y el IPGRI (2001), en que se menciona que la mujer ha influido en la evolución del maíz y en su alta variabilidad, generadas al incidir en el proceso de selección y conservación de las semillas.

Por otro lado, existen datos alusivos a la diferencia de gustos entre hombres y mujeres al momento de seleccionar los maíces; por ejemplo, Ortega-Paczka (2003), menciona que:

« aunque existen grandes variaciones en distintos grupos humanos, los varones son los que en general se ocupan de tener poblaciones que se adapten bien a las condiciones de su campo y a las demandas del mercado. Las mujeres prefieren maíces con alta calidad culinaria y fáciles de desgranar y exigen que además de los maíces blancos para tortillas comunes se cultiven los azules y rojos para antojitos, los amarillos para los animales y otras variantes ».

21 En otro estudio, realizado en el Valle Central de Oaxaca (Oax.) por Bellon et al. (2000), se encontraron varias diferencias en los patrones de votación entre hombres y mujeres al momento de elegir los maíces que son cultivados, además de que las mujeres en promedio escogieron una mayor cantidad de razas en comparación con la elección de los hombres (doce y nueve respectivamente).

Figura 3 : Maíz desgranado y semillas seleccionadas para distintos propósitos

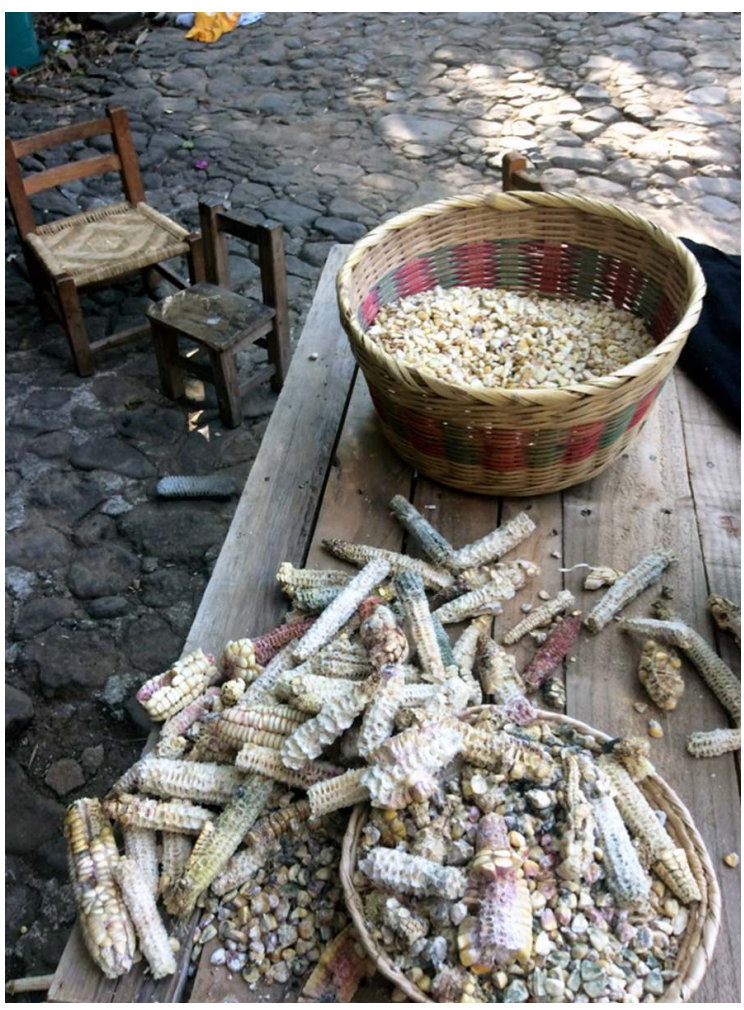

FOTOgRAFÍA DE E. L. HAgMAN AgUILAR

\section{Los rituales}

22 Antes de hablar del quehacer sobresaliente de las mujeres en los rituales, cabe destacar cómo visualizan su semilla: 
- Sra. Irene: « Significa mucho... es la vida ».

- Sra. Amelia: « es el alimento, que si no comemos, nos morimos, se debe cuidar el maicito y todo lo que se come. Si no se cuidara, se perdería el maíz, nos moriríamos porque estamos acostumbrados a comer la tortillita ».

- Sra. Sabina: «Es sagrado, sino es por él no vivimos, no comemos. Es bueno tener y sembrar porque si no tenemos, siento que no tengo nada. Las personas han perdido sus semillas por sus gustos, a mí no me gustaría perderlo, es importante ».

- Sra. Dionisia: « Nosotros sembramos al maíz criollo que desde mis abuelos lo van sembrando, porque las tortillas están mejor, aunque -sea- una tortilla con sal; y ahora que hay maíz hibrido es diferente, ya tiene otro sabor, nosotros no sembramos hibrido. Es sagrado porque es nuestra vida, nuestro alimento, comemos el maíz que cultivamos ».

Estas respuestas involucran lo que Beutler (1973) denomina como un "animismo» expresado en los diminutivos afectivos y en los « conjuros » elaborados antes, durante y después de la siembra evocando respeto, solemnidad, alabanza y bienaventuranza para el «maicito». A continuación mencionamos algunos de los rituales que forman parte del bagaje cultural amatleco en torno al maíz, destacando los dos últimos debido a que las mujeres son quienes los realizan de manera casi exclusiva y por sus implicaciones sociales y culturales.

\section{La bendición de la semilla:}

Es una actividad en la que suelen concurrir las mujeres en compañía de sus hijas e hijos y de su esposo (Figura 4); la bendición/energización la realizan en la iglesia o en algún terreno, ahí forman un altar en el que colocan parte de las semillas para la siembra, tanto del maíz como del frijol y de la calabaza. Luego de la ceremonia, llevan las semillas a su casa donde se revuelven con el resto. Ya para cuando " va a salir al campo el maicito », lo ponen en el altar de la casa y la mujer lo sahúma.

\section{La Sra. Irene narra:}

« Íbamos y sacábamos la semilla, decía mi papá: ya vamos a sembrar mañana; entonces ya preparábamos la semilla, lo poníamos ahí enfrente del altar el maíz desgranado y le echábamos su sahumerio. El día que ya se va a sembrar se lleva y allá se le platica al maicito, se le dice: ya te venimos a dejar en el campo para sembrarte para nuestro alimento y ya vienes a sufrir todo esto, sufres el aire, sufres el calor, sufres que un animalito ya te vino a comer. Todo eso sufre el maíz porque es nuestra vida; ya que está más grandecito ya sufre que no llueve a veces, eso también lo pone triste, cuando no llueve le molesta mucho el gusano, el cogollito; cuando ya viene saliendo el jilote -(del náhuatl xilotl: infrutescencia de la planta de maíz que no ha madurado)- ahí se mete y lo come y lo pierde, por eso le platicamos al maicito que todo sufre». 
Figura 4 : Sra. Irene Ramírez Cásares colocando flores en el altar para la bendición de las semillas el 15 de mayo, día de San Isidro Labrador

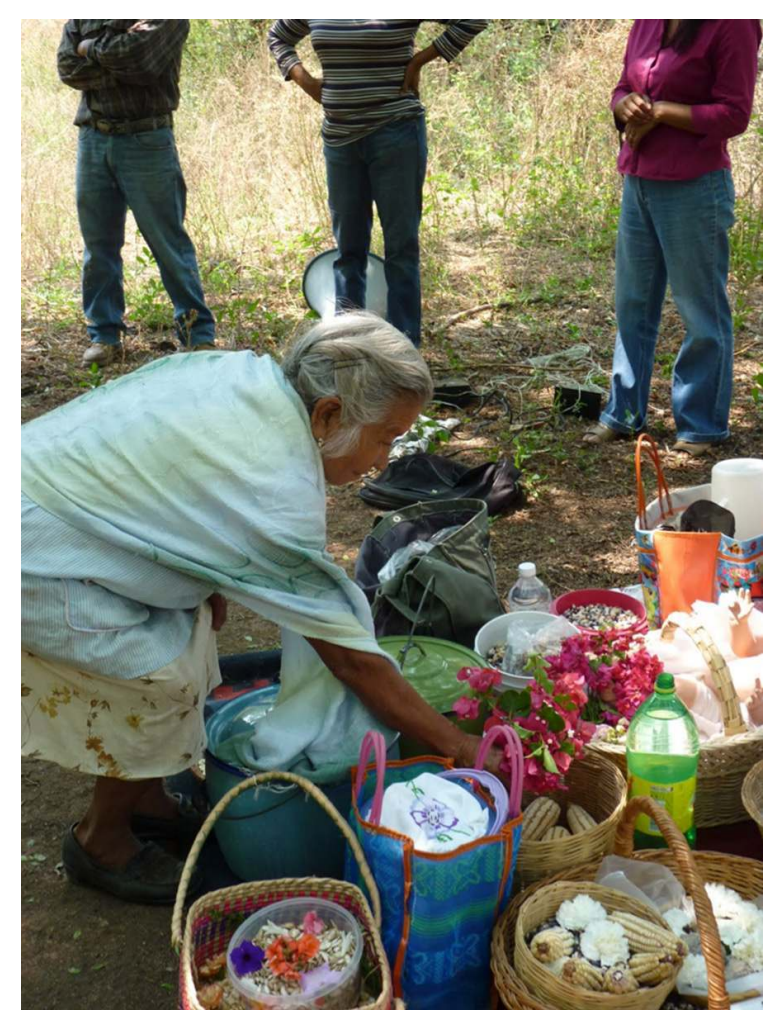

FOTOgRAFÍA DE E. L. HAgMAN AgUILAR

\section{La cruz de yautli}

Aunque puede hacerlo toda la familia, hay casos en los que son las mujeres quienes van a recolectar las flores del «pericón» o yautli (Tagetes lucida Cav.) empleadas en la elaboración de "cruces prehispánicas" (cruz equidistante que indica los cuatro puntos cardinales) llevadas a bendecir a la iglesia para después ser colocadas en las milpas, las puertas y las ventanas de las casas el día del «periconazo» o «elotiza» (28 de septiembre), una celebración ritual en la que se cosechan los primeros elotes tiernos (Figura 5).

Cabe mencionar que el 29 de septiembre se dedica a San Miguel Arcángel. En el vecino barrio de San Miguel, en Tepoztlán, la iglesia es adornada con flores de yautli, la gente de la cabecera municipal aprovecha para llevar a bendecir sus flores y «cruces prehispánicas »; esto evidencia la articulación e incluso la superposición de la religión católica sobre la tradición náhuatl en la que se utiliza dicha flor desde tiempos prehispánicos. A este respecto, la señora Rosalinda comenta: « ... hacen -hacemos- la cruz del pericón para el campo y la casa, protegen las milpas de que San Miguel Arcángel corre entre las milpas». 
Figura 5 : Flores del « pericón » O YAUTLI (Tagetes lucida Cav.)

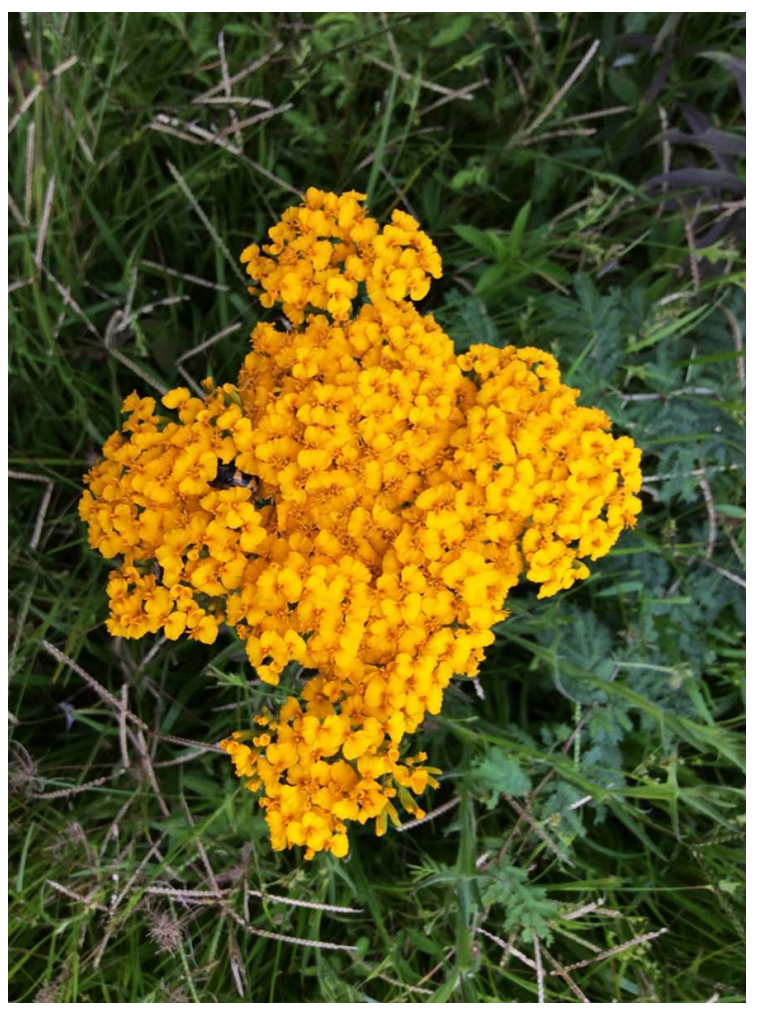

FOTOgRAFíA DE E. L. HAgMAN AgUILAR.

\section{El recibimiento del maíz}

La jefa de familia recibe al maíz recién cosechado, lo sahúma dándole la bienvenida con gusto porque «regresó a casa » para alimentar a la familia, ese día hacen una comida especial para los que fueron a la pixca (Figura 6). 
Figura 6 : Sra. Irene sahumando las mazorcas cosechadas para recibirlas en su casa

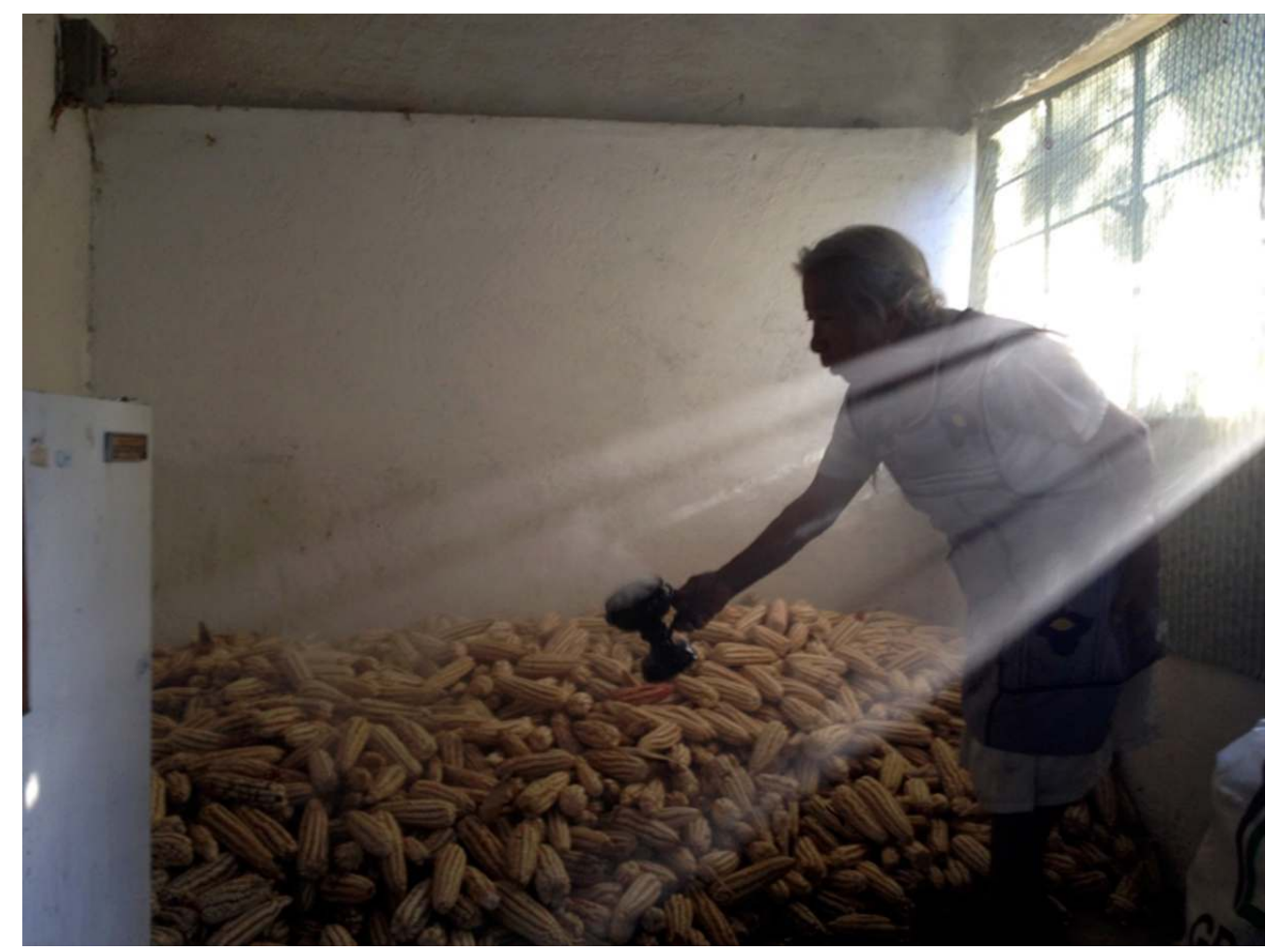

FOTOgRAFíA DE J. C. GONZÁLEZ RODRÍgUEZ

29 La razón por la que lo recibe la mujer es explicada por la joven Leticia Corrales, hija de la Sra. Dionisia:

« es porque la mujer lo va a administrar, es la que tiene más contacto con los alimentos... la mujer es la fertilidad y como la madre tierra está dando sus frutos pues es la que tiene que recibir, mi abuelita nunca nos lo dijo, pero tenía que ser la mujer más grande, como de respeto, es la administradora. El hombre lo trae, es el intercambio de la dualidad ";

su mamá, a su vez, nos compartió lo siguiente

«a mis niñas les enseñamos a querer a la madre tierra que nos da de comer. Nosotros en la familia desde niños, les enseñamos que al campo hay que quererlo y respetarlo; vienen personas para que les vendamos un terreno, no se vende, porque son tierras de los abuelos que sembraban maíz, frijol, que lo cultivaban, y así nosotros hay que seguir sembrándolas para poder tener nuestro propio maíz ».

31 Estas palabras corroboran la transferencia de conocimientos madre-hija que ocurre en Amatlán, observada también por Román-Montes de Oca y Guzmán-Gómez (2013) que mencionan que hay una incorporación de las nuevas generaciones en las tareas agrícolas y las ceremonias.

32 Éste ritual entraña, por un lado, el simbolismo prehispánico de la feminidad como símbolo de fertilidad y por otro, que la mujer es tradicionalmente una guardiana de las semillas de maíz, al fungir como administradora del sustento, suceso constatado por la etnografía actual (FAO-IPGRI, 2001), la alusión de la mujer hacia la fertilidad y la milpa está relacionada a su vez, con dos cuestiones:

La primera es que, de acuerdo con López-Austin (1998), en el mundo y el pensamiento prehispánico, todo lo existente era una mezcla de esencias de lo masculino y lo femenino 
y era el predominio de una de ellas lo que determinaba la clasificación y el grado de pertenencia de cada ser a uno de los campos taxonómicos; en ese sentido, el periodo de lluvias era de « dominio » femenino, momento en el que se unían las semillas con el agua y las fuerzas de las diosas productoras, dando origen a nuevas plantas.

La segunda, es que existe un gran número de deidades femeninas mesoamericanas. En el caso de aquellas veneradas por los mexicas, destacan Chicomecoatl, que representa el mantenimiento, la subsistencia y la fertilidad del maíz como fruto, y Xilonen, que representa los jilotes y el maíz tierno, ambas relacionadas con la agricultura, así como Chalchiuhtlicue, que representa el agua que ya está en la tierra, la de los ríos y los lagos, que tenía una función fertilizadora (López-Arenas 2006). A este respecto la Sra. Sabina complementa: «La tierra es de una madre que es en la que estamos, en ella, la tierra madre ».

\section{La organización de los festejos}

La intervención de las mujeres amatlecas en los festejos y ceremonias, no se limita sólo a la preparación de los alimentos adecuados para cada ocasión, como se verá más adelante, sino que funge como gestora, organizadora y supervisora para llevar a buen término cada evento. Ejemplo de ello es que «la mujer de la casa » es la primera en levantarse y la última en dormirse durante la fiesta patronal, dando indicaciones durante todo el proceso a los demás miembros de la familia que participan de manera activa para recibir y atender al gran número de invitados (de 100 hasta 300 en cada casa).

La organización de las festividades implica -además de eminentes esfuerzos y destrezas con entrega y dedicación- el invaluable deber de transmitir y socializar los conocimientos y los valores imbricados en estos actos hacia las personas que coadyuvan en su realización (infantes, adolescentes y adultos). El hecho de que las mujeres funjan como organizadoras de los eventos y administradoras del sustento, fue registrado también en el estudio realizado en la comunidad por Román-Montes de Oca (2013), quien menciona que estas representaciones de la mujer son muy valoradas dentro del seno familiar, siendo escuchadas y consideradas para la toma de decisiones, sin que esto necesariamente modifique las bases de la organización familiar ni los roles que cada integrante debe cumplir.

\section{Los procesos culinarios}

37 En cada hogar, son las mujeres las responsables de la preparación de los alimentos, elaborados con productos obtenidos en la milpa, en los huertos de traspatio, en el « monte » o comprados en diversos sitios de la región, como Tepoztlán o Cuautla. En el caso del maíz, el resultado final de su trasformación va a depender del grado de maduración de la mazorca

\section{Con los elotes}

Cuando el fruto está « duro » y « de buen tamaño » quiere decir que es buen tiempo para cortar los elotes, esto ocurre a finales de septiembre y entonces ponen la « lumbre » en el campo y los comen enteros, hervidos o asados; también hacen « esquites " (son los granos de elote hervidos en agua con sal, epazote Dysphania ambrosioides (L.) Mosyakin \& Clemants, u otras « hierbas de olor » y a veces con patas de pollo), atole, "chileatole » 
(con chile serrano Capsicum annuum L. y epazote), tamales y sopa; o bien los guisan con calabazas, epazote y crema; otra forma es con flor de calabaza, con jitomate (Solanum lycopersicum L.), con chayote (Sechium edule (Jacq.) Sw.) o con verdolaga (Portulaca oleracea L.).

\section{Con las « camaguas »}

Cuando el fruto comienza a secarse y la hoja se pone "amarilla», es el momento de aprovechar las camaguas. Se utiliza principalmente para preparar los "tlaxcales" (muelen los granos sin agua, le ponen azúcar, canela, leche, queso, huevo y mantequilla, aunque también pueden ser salados, preparados con frijoles; se moldean en forma de triángulo y se fríen en el comal), que se acostumbran en octubre y noviembre, tradicionalmente para ofrendarlo el día de los difuntos. Otras formas de comerlas es asar el fruto, preparar esquites, tamales o atole (la masa, no es nixtamalizada, remojan los granos por dos días y los llevan a moler, luego la ponen en una olla para cocerla con canela y se le puede agregar leche).

\section{Con los granos maduros}

40 Su preparación depende del color de la infrutescencia. Los granos azules se usan para hacer tortillas y atole caliente o fresco (sin leche, se le añade canela y ayuda para el tratamiento del riñón). Los rojos son para hacer tortillas, pero lo más común es el atole de champurrado (se tuesta el maíz, se lleva a moler con la canela, se le pone el chocolate, se remoja y ya molido se cuela), aunque igualmente lo pueden hacer con los elotes blancos o azules.

Por último, los frutos blancos se emplean para elaborar atoles, tortillas, tamales, «tlacoyos", « sopes ", quesadillas, tacos, «picaditas», « itacates », entre otros, los dos últimos son propios de la gastronomía morelense (las picaditas son para el almuerzo, es una especie de "sope » al que se le pone salsa roja o verde, crema y encima queso. Los «itacates", son como "gorditas» triangulares, que se fríen en manteca de cerdo, se rellenan de queso, crema y salsa, aunque pueden llevar algún guiso). Para preparar estos platillos es necesaria la nixtamalización. De acuerdo con el saber popular, el nixtamalizado del maíz se hace para que los granos se puedan moler, se le echa la cal para que los ponga suaves, por ejemplo, la Sra. Sabina dice que: «sino se pone tieso. La cascarita está tiesa si no se le echa cal ». Por otro lado, la señora Irene, transmite su conocimiento a todo aquel que esté interesado en aprovecharlo, enseñando el proceso de la nixtamalización, es por eso que este saber sigue vivo y se extiende ${ }^{2}$.

Para trasformar la masa nixtamalizada en tortillas es necesaria la molienda y la cocción. La molienda de la mezcla nixtamalizada se realiza en alguno de los molinos eléctricos que hay en el pueblo, ahí les cobran por kilo, anteriormente lo «martajaban » (moler) en el " metate ${ }^{3}$ con su «metlapil », las campesinas mencionan que el «metate» le daba más sabor a la masa y que sienten que entonces era más nutritiva, pero ya se «modernizaron" y por eso lo hacen ahora en el molino con el que se invierten menos tiempo y esfuerzo físico. Con la masa nixtamalizada y molida van haciendo bolitas que aplastan a mano o con la «tortilladora » (prensa metálica con la que mecánicamente se aplana la masa para hacer tortillas); según recuerda la señora Amelia « la tortilladora se usa en Amatlán desde hace aproximadamente 40 años ». La forma en que echan las tortillas al comal, depende de cómo se acomoda cada una, ya sea que la avienten con la 
palma de la mano hacia abajo o la dejen caer muy despacio, deslizando la palma de la mano hacia un lado (Figura 7).

Figura 7 : Sra. Maximina Guerrero echando tortillas en estufa ahorradora de leña

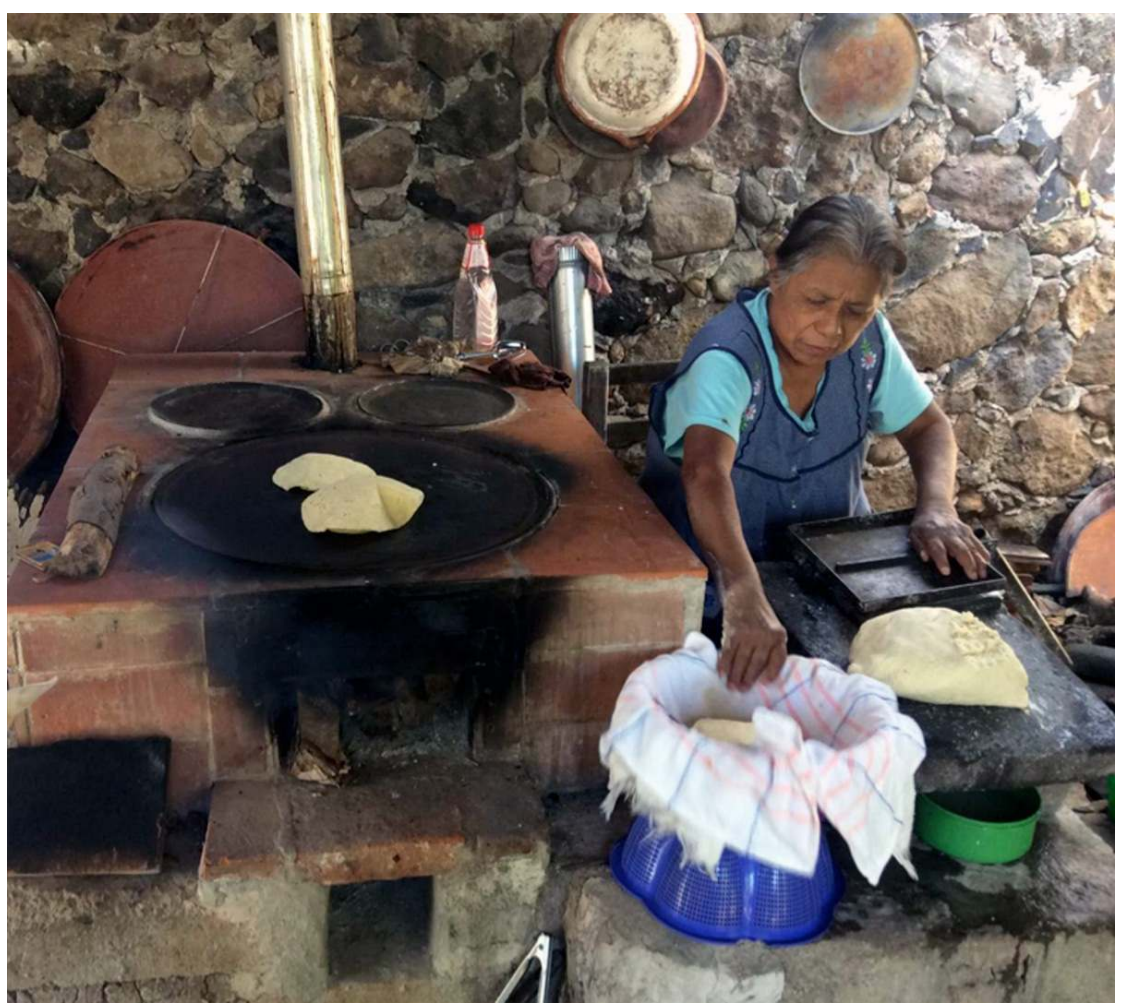

FOTOgRAFÍA DE E. L. HAgMAN AgUILAR. (¿NO SE PUEDE AÑADIR QUE EN LA IZQUIERDA ESTÁ EL COMAL EN QUE CUECEN TORTILLAS?)

El acomodo en el « tortillero » (recipiente con tapa en el que se colocan las tortillas recién hechas envueltas en una servilleta de algodón) es algo especial, pues dicen que la tortilla tiene « cara » (que es la que se cuece primero) y « espalda " (la parte que se cuece cuando se voltea la tortilla en el comal); algunas mujeres tienen la costumbre de colocar la primera tortilla con la « cara » hacia arriba y el resto de " espalda », otras las colocan al revés; ambas formas tienen como fundamento la creencia de que si no lo hacen de tal manera, las personas que coman de esa tanda de tortillas quedarán con hambre. Otra práctica es que la primera y la última tortilla que se pongan en el tortillero deben persignarse, para que, según la señora Sabina, « no coman del otro, sino de diosito, si no se bendice es como si adorara al de -los- cuernos ».

El maíz siempre está presente en alguna de sus formas gastronómicas y en todas y cada una de las celebraciones que se realizan en el pueblo, ya sean tradicionales, religiosas o creadas recientemente.

Para empezar, las tortillas no sólo forman parte de su dieta habitual, sino que nunca faltan en las festividades para acompañar los guisos propios del día conmemorado, además en el «día de Quetzalcóatl», en el "festival del maíz» y en el «festival de la medicina tradicional», es común que los amatlecos coloquen puestos donde venden antojitos mexicanos hechos con maíz. 
Los «tlaxcales », son parte de las ofrendas del 1 y 2 de noviembre, pero también hay quienes los preparan pasados ocho días de esas fechas y entonces los colocan en la ofrenda junto a una taza de café.

7 Los tamales suelen elaborarlos para navidad, año nuevo, el día de Santa María Magdalena (la patrona del pueblo), el día de los santos inocentes o de todos los santos, o bien en los cumpleaños y los santos familiares. Hay diferentes tipos de tamales: los de frijol tierno o seco, los de carne de pollo o de puerco, los de salsa verde o roja, los de flor de colorín ( Erythrina coralloides DC) (cuando es temporada, de diciembre a marzo) y los dulces (con canela y azúcar).

El pozole lo hacen cuando hay una fiesta de santo o cumpleaños, le ponen el grano del maíz ancho blanco que es el que siembran y lo preparan con pollo, carne de puerco o más recientemente con setas y puede ser blanco o rojo.

9 La mujer, es la encargada de preparar los alimentos para cada jornal, la comida que suelen preparar no es específica, llevan « lo que haya », algunos ejemplos son: la sopa de pasta, los frijoles, el caldo de pollo, la salsa con yemas de huevo, la sopa de arroz y en época de lluvias los "quintoniles" (Amaranthus hybridus L.) o "yerbitas de campo " guisados, la flor de calabaza, las calabacitas tiernitas con jitomate o secas, la ensalada de hongo de «cazahuate ", Pleurotus ostreatus (Jacq. : Fr.) P. Kumm., y por supuesto lo que nunca puede faltar son las tortillas. Cuando terminan la labor agrícola de la «segunda mano ", hacen especialmente mole verde y con las hojas de la milpa hacen tamales de frijoles y de masa, esto es para dar gracias de que ya se terminó la labor en el campo, a esto le llaman la « despachada ».

Es necesario destacar que las mujeres amatlecas son depositarias y salvaguardas de la cocina regional (Figura 8), a la que Padilla (2006) se refiere como « patrimonio intangible de las comunidades, que se distingue por su capacidad de evocar valores, sabores, modos, estilos y sazones "; y que tiene al maíz como eje vertebral que deviene como un elemento indispensable para su mantenimiento y que está bajo amenaza, hecho que se manifiesta a escala nacional (CONACULTA 2004). 
Figura 8 : El tradicional mole preparado para la fiesta de Santa María Magdalena, la Patrona del pueblo de Amatlán de Quetzalcóatl

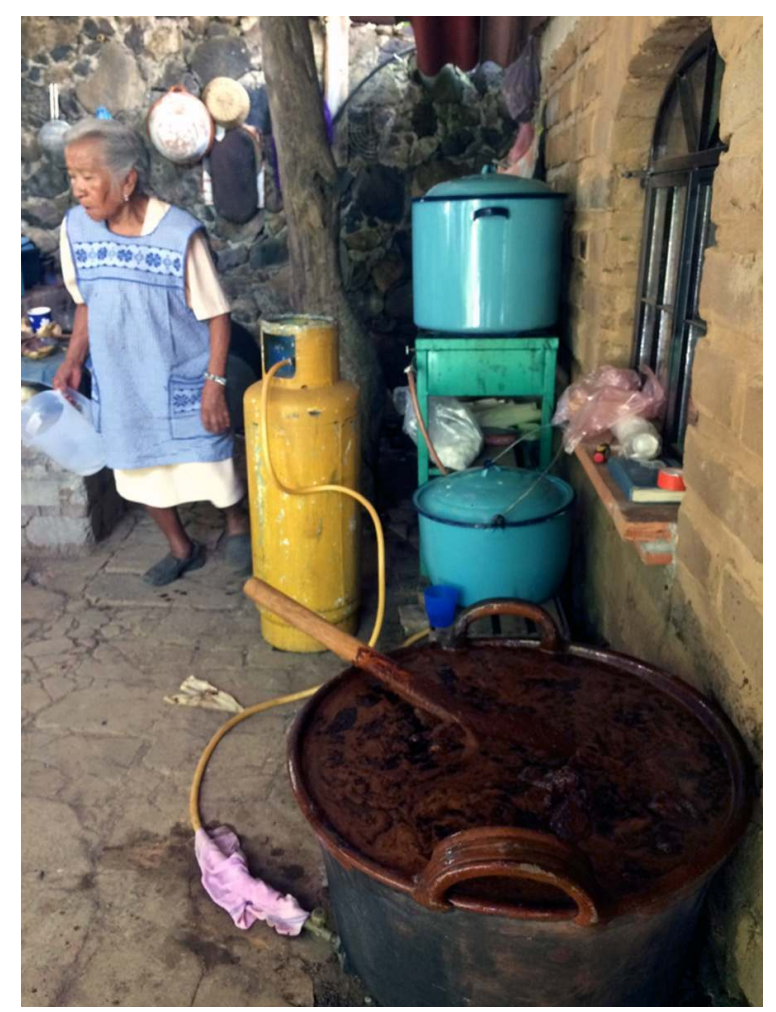

FOTOgRAFÍA de R. RAMÍREZ GUERRERO. (¿NO TIENES LA RECETA DESTA PREPARACIÓN COMPLEJA Y PRESTIgIOSA?) alimentos tanto para la vida diaria, como para las festividades, los rituales, las ceremonias y las ofrendas; b) al entrevistar a las mujeres, que con amor y devoción llevan a cabo los procesos culinarios de transformación del maíz en sus diferentes etapas fenológicas (elote tierno, camagua y mazorca), con la guía de sus tías y abuelas y con la ayuda de sus hijas, nueras y nietas; c) al degustar los diversos platillos con productos de la milpa que en su sabor y en su aroma, particularmente deliciosos, se asoman la dedicación y el valor que representan para las familias amatlecas; d) al aprender el proceso de nixtamalización y la 
elaboración de tortillas con la amable y paciente enseñanza de la Sra. Irene Ramírez Casares; y e) al asistir a las ceremonias, rituales y festejos a lo largo del ciclo de cultivo, en los que los alimentos son un elemento esencial.

\section{Conclusiones}

La milpa es un sistema en el que coevolucionaron las plantas que lo conforman y las culturas que le dieron vida, es un bien común, fuente de diversidad, bienestar y nutritivo sustento; es un espacio cargado de significados materiales e inmateriales; es una y a la vez muchas culturas vivas colmadas de identidades y de convicciones; es el lugar en el que germinan y crecen los diferentes maíces nativos, base de la alimentación de los mexicanos y materia prima para la elaboración de los platillos que componen su rica gastronomía valorada a nivel mundial. Para los campesinos que siguen sembrando el maíz nativo en Amatlán, es una práctica vital en la que hombres y mujeres tienen un papel que se complementa, se valora y se transmite de una generación a otra; todo ello ocurre en el día a día dentro del seno familiar, en la que los padres heredan en vida a sus hijos con los conocimientos, las creencias, los rituales y las técnicas.

Es claro que en ésta comunidad la mujer juega un papel fundamental en la reproducción del maíz y sus prácticas culturales, los resultados aquí expuestos develan que éste hecho se debe esencialmente a cuatro razones: 1) por su colaboración dinámica en las labores de la milpa; 2) por su especial participación en los rituales y su figura primordial en la cosmovisión prehispánica; 3) por su rol cardinal en la organización familiar; y 4) por ser depositaria y salvaguarda del patrimonio cultural de la gastronomía morelense, que depende a su vez de la conservación del maíz nativo y su diversidad.

El legado culinario que llevan a cuesta cotidianamente, son los valores y los conocimientos aplicados en el manejo de la milpa que portan y transmiten al resto de los miembros de la familia; la gestión de los diferentes festejos a lo largo del calendario ritual agrícola (en los que por supuesto el maíz nativo forma parte de su esencia), es así como, su participación en el proceso de selección de las semillas y por tanto en su diversificación; son algunos de los hechos que revelan que en efecto, las mujeres amatlecas son esenciales para la conservación dinámica del patrimonio biocultural que representan el maíz nativo y la milpa tradicional.

A las familias amatlecas que siguen haciendo milpa. Al grupo de medicina tradicional Atekokolli. A la comunidad de Amatlán de Quetzalcóatl, Tepoztlán, Morelos. Al laboratorio de Etnobotánica de la Faculta de Ciencias, Universidad Nacional Autónoma de México.

\section{BIBLIOGRAPHY}

Alexiades M. N. 1996 - Collecting ethnobotanical data: An introduction to basic concepts and techniques. In : Alexiades M. N. (Ed.) Selected Guidelines for Ethnobotanical Research: A Field Manual. EE. UU. The New York Botanical Garden : 53-94. 
Alvarado F. 1992 - Ce-Acatl Topiltzin Quetzalcoatl. Folleto. Vol. 1. México, D. F. 31 p.

Bisquerra R. 1996 - Métodos de Investigación Educativa. Guía Práctica. Barcelona, CEAC, 400 p.

Bellon M. R., Smale M., Aguirre A., Taba S., Aragón F., Díaz J. \& Castro H. 2000 - Identifying Appropriate Germplasm for Participatory Breeding: An Example from the Central Valleys of Oaxaca, Mexico. México, D. F. CIMMYT Economics Working Paper 00-03. 14 p.

Beutler G. 1973 - Algunas Oraciones y Ceremonias Relacionadas con el Cultivo del Maíz en México. Indiana 1 : 93-112.

COA 2012 - El maíz no es una Cosa, es un Centro de Origen. México, D. F. Colectivo por la Autonomía, $317 \mathrm{p}$.

CONABIO 2012 - Portal de Geoinformación. Sistema Nacional de Información sobre Biodiversidad. Comisión Nacional para el Conocimiento y Uso de la Biodiversidad. Disponible en ligne : http:// www.conabio.gob.mx/informacion/gis/

CONACUlTA 2004 - Pueblo de Maíz. La Cocina Ancestral de México. México, D. F. Consejo Nacional para la Cultura y las Artes, $111 \mathrm{p}$.

Corneli J. 2005 - Maíz: Fuente de Identidad y Presente de un Pueblo. Tesis Profesional. Facultad de Psicología. México, D. F. Universidad Nacional Autónoma de México, 157 p.

FAO-IPGRI 2001 - El Papel de la Mujer en la Conservación de los Recursos Genéticos del Maíz. Guatemala. Roma, Organización de las Naciones Unidas para la Agricultura y la Alimentación, Instituto Internacional para los Recursos Fitogenéticos, $56 \mathrm{p}$.

Giard L. 1999 - Hacer de comer. In : De Certeau M., Giard L. \&. Mayol P. La Invención de lo Cotidiano II. Habitar, Cocinar. México, D. F. Universidad Iberoamericana : 151-256.

Gispert M., Diego N., Jiménez J., Gómez A., Quintanilla J. M. \& García L. 1979 - Un nuevo Enfoque en la Metodología Etnobotánica en México. Medicina Tradicional 2 (7) : 41-52.

Gispert M., Rodríguez H., Coutiño B., Díaz A. \& Peralta S. 2007 - Creación de un Jardín Etnobotánico en el recinto del Atekokolli de Amatlán de Quetzalcóatl, Morelos. México, D. F. Imprenta Guevara, 15 p.

INEGI 2010 - Censo de Población y Vivienda. Principales resultados por localidad. México, D. F. Instituto Nacional de Estadística, Geografía e Informática.

Kato Y. A., Ortega-Paczka R., Boege E., Wegier A., Serratos-Hernández J. A., Alavez V., JardónBarbolla L., Moyers L. \& Ortega-Del Vecchyo D. 2013 - Origen y diversidad del maíz. In : ÁlvarezBuylla E. \& Piñeyro A. (Ed.) El Maíz en Peligro ante los Transgénicos. Un Análisis Integral Sobre el Caso de México. México, D. F. Universidad Nacional Autónoma De México. México : 25-70.

López-Arenas G. 2006 - Deidades de la Fertilidad Agrícola en el Panteón Mexica. Estudios Mesoamericanos 7 : 45-52.

López-Austin A. 1998 - La Parte Femenina de Cosmos. Arqueología Mexicana 5 (29) :6-13.

López-García J. \& Oliver-Guadarrama R. 2010 - Suelos. In : Bonilla-Barbosa J. R., Mora V. M., LunaFigueroa J., Colín H. \& Santillán-Alarcón S (Ed.) - Biodiversidad, Conservación y Manejo en el Corredor Biológico Chichináutzin. Condiciones Actuales y perspectivas. Cuernavaca. Universidad Autónoma del Estado de Morelos : 21-32.

Ortega-Paczka R. 2003 - La diversidad del maíz en México. In : Esteva G. \& Marielle C. (Ed.) Sin Maíz no Hay País. México, D. F. Consejo Nacional para la Cultura y las Artes, Museo Nacional de Culturas Populares : 123-154. 
Padilla C. 2006 - Las Cocinas Regionales. Principio y Fundamento Etnográfico. Ponencia para el VII Congreso Internacional de Sociología Rural. Quito, Ecuador 20 -24 de noviembre de 2006.

Disponible en: http://www.academia.edu/1938413/

Las_cocinas_regionales._Principio_y_fundamento_etnografico

Rodríguez G., Gil J. \& García E. 1999 - Metodología de la Investigación Cualitativa. Malaga. Ediciones Aljibe : 62-77.

Román-Montes de Oca E. 2013 - El Maíz en la Vida Cotidiana de las Familias Campesinas de Amatlán de Quetzalcóatl. Tesis Doctoral. Cuernavaca. Universidad Autónoma del Estado de Morelos, Facultad de Ciencias Agropecuarias, $191 \mathrm{p}$.

Roman-Montes de Oca E. \& Guzmán-Gómez E. 2013 - Mujer, trabajo y persistencia del maíz. Revista de Estudios de Género. La ventana 4 (38) : 164-211.

Taylor S. J. \& Bogdan R. 1987 - Introducción a los Métodos Cualitativos de Investigación. Barcelona, Paidós Ibérica, 345 p.

\section{NOTES}

1. Forma antigua de cultivar la milpa que consistía en podar los árboles grandes y en cortar las yerbas, los arbustos y los árboles pequeños en un terreno; para sembrar se juntaba la tierra en montones con aproximadamente $70 \mathrm{~cm}$ de separación, en ellos se colocaban las semillas de maíz, frijol y calabaza; cuando el maíz alcanzaba los 30 y los $60 \mathrm{~cm}$ de altura se desyerba el terreno nuevamente y se volvían a levantar los montones de tierra en dos momentos, no se utilizaba ningún tipo de abono ajeno al propio terreno.

2. La nixtamalización del maíz, es decir cocer los granos con cal, además de ablandar esos granos, libera la niacine, o vitámine B3 que protege contra la pelagre, grave carencia que es mortal. Se llama también vitámine PP que significa Pelagre preventiva (nota del editor).

3. Metate, utensilio hecho con una piedra volcánica rectangular y espesa, tripode, sobre la cual se muelen los granos de maíz con el metlapil, « hijo del metate», pieza redonda e irregular del mismo material (nota del ed.).

\section{ABSTRACTS}

The mexican maize (Zea mays L.) preserved in Amatlán de Quetzalcóatl (Tepoztlán, Morelos) is an example of a dynamic biocultural conservation. The inhabitants of this community are nahuas, an ethnic group that is in resistance facing the loss of their traditions, culture and lineage. The community is located in a region of high biodiversity. In this study we pursue to identify the cultural, environmental and socio-economic factors that have contributed to the preservation of native maize in this community. Women of Amatlán had an equal participation in crop management and also played several special roles as the harvest reception at home, cooking and carrying food to the agricultural field; moreover they're the sustenance administrator of their homes and creators of culinary transformation processes of milpa's crop species. 
La persistencia del maíz nativo (Zea mays L.) en Amatlán de Quetzalcóatl (Tepoztlán, Morelos) es un ejemplo de conservación biocultural dinámica; sus habitantes pertenecen a un grupo étnico que se resiste a perder sus tradiciones, su cultura y su linaje. La comunidad está en una región biodiversa. La mujer amatleca participa de manera igualitaria en el manejo del cultivo, pero posee un papel especial en tradiciones como el recibimiento en la casa del maíz cosechado; además cocina y lleva los alimentos al campo; también es administradora del sustento y creadora de los procesos de transformación culinaria de los productos de la milpa.

La persistance du maïs indigène (Zea mays L.) à Amatlán de Quetzalcóatl (Tepoztlán, Morelos) est un exemple de conservation bioculturelle dynamique; ses habitants appartiennent à un groupe ethnique, les nahuas, qui refuse de perdre ses traditions, sa culture et sa lignée. La communauté vit dans une région riche en biodiversité. Les femmes participent à la gestion des cultures et lorsqu'il est récolté, le maïs est soumis à des rituels particuliers quand on l'emmagasine dans la maison Elles le cuisinent et portent les repas sur le terrain; elles administrent ainsi la subsistance de chaque famille et les processus de création culinaire dans le traitement des produits de la « milpa ».

\section{INDEX}

Mots-clés: maïs natif, conservation bioculturelle, femme

Keywords: Mexican native maize, biocultural conservation, woman

Palabras claves: maíz nativo, conservación biocultural, mujer

\section{AUTHORS}

\section{ERICA HAGMAN AGUILAR}

Maestría en Ciencias de la Sostenibilidad, Universidad Nacional Autónoma de México

\section{MONTSERRAT GISPERT CRUELLS}

Facultad de Ciencias, Universidad Nacional Autónoma de México 